\title{
Overview of Pheochromocytomas at Vilnius University Hospital Santariskiu Clinics (VUHSC): 5 year results
}

Milda Girdziute ${ }^{1}$, Zydrune Visockiene ${ }^{12}$.

1 Vilnius University

2 Vilnius University Hospital Santariskiu Klinikos

\section{Introduction}

According to various guidelines, all patients with adrenal masses must be checked for pheochromocytoma because up to $15 \%$ of patients with pheochromocytomas have no history of hypertension. The incidence of pheochromocytoma in clinical studies ranges from 1.5 to $14 \%$ and it is crucial to confirm the diagnosis before surgery because of life threatening complications.

\section{Aim}

To estimate the prevalence and describe diagnosis, treatment and follow up strategy of pheochromocytomas at VUHSC.

\section{Methodology}

Cases coded as D35.0; D44.1; E27.0; E27.5; E27.8; E27.9 according to ICD-10 classification were retrieved from database. Electronic data capture system was used to collect information.

\section{Results}

\section{2 patients with adrenal masses}

$\mathbf{5 0 \%}$ screened for pheochromocytomas

$24(3.4 \%)$

pheochromocytomas

\section{Patient characteristics:}

\begin{tabular}{|l|l|}
\hline Males & $5(20.8 \%)$ \\
\hline Females & $19(79.2 \%)$ \\
\hline Age & $55 \quad 11.5$ years \\
\hline Symptoms & $21(87.5 \%)$ \\
\hline Incidentally found & $15(62.5 \%)$ \\
\hline
\end{tabular}

Tumour characteristics:

\begin{tabular}{|l|l|}
\hline Size & $48.0 \quad 23,4 \mathrm{~mm}$ \\
\hline $\begin{array}{l}\text { Attenuation value on noncontrast } \\
\text { computed tomography }\end{array}$ & $33.7 \quad 11.6 \mathrm{HV}$ \\
\hline Side: & \multicolumn{2}{|l|}{} \\
\hline -Right & $8(33.3 \%)$ \\
\hline -Left & $15(62.5 \%)$ \\
\hline -Bilateral & $1(4.2 \%)$ \\
\hline
\end{tabular}

Hormonal evaluation:

\begin{tabular}{|l|l|}
\hline Performed & $20(83.3 \%)$ \\
\hline $\begin{array}{l}\text { Isolated elevations of either } \\
\text { adrenaline or metanephrine }\end{array}$ & $4(16.7 \%)$ \\
\hline $\begin{array}{l}\text { Isolated elevations of either } \\
\text { noradrenaline or normetanephrine }\end{array}$ & $3(12.5 \%)$ \\
\hline
\end{tabular}

Genetic syndromes:

$2(8,3 \%)$ subjects:

-Von Hippel Lindau syndrome

- Type 1 neurofibromatosis

\section{Symptoms of pheochromocytoma:}

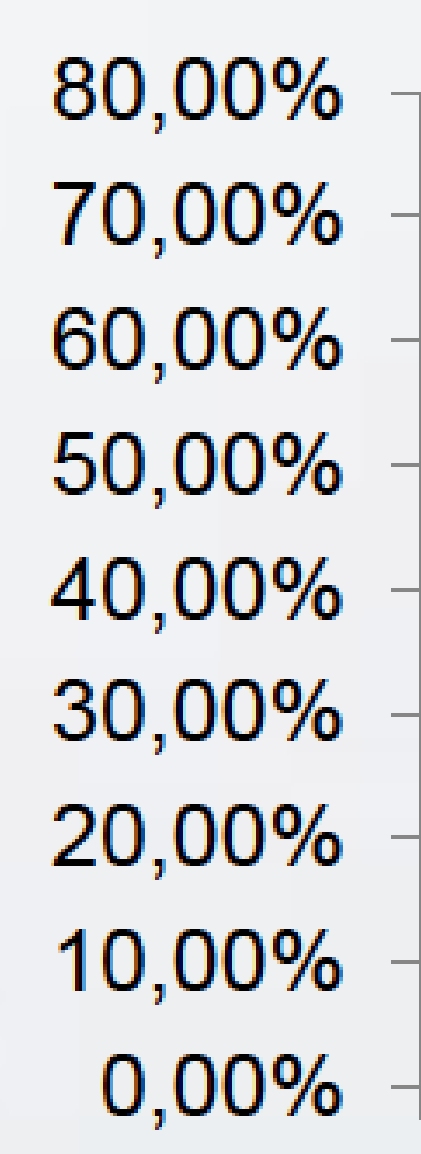

$70,00 \%$

$60,00 \%$

$50,00 \%$

$40,00 \%$

$30,00 \%$

$10,00 \%$

$0,00 \%$

Hypertension
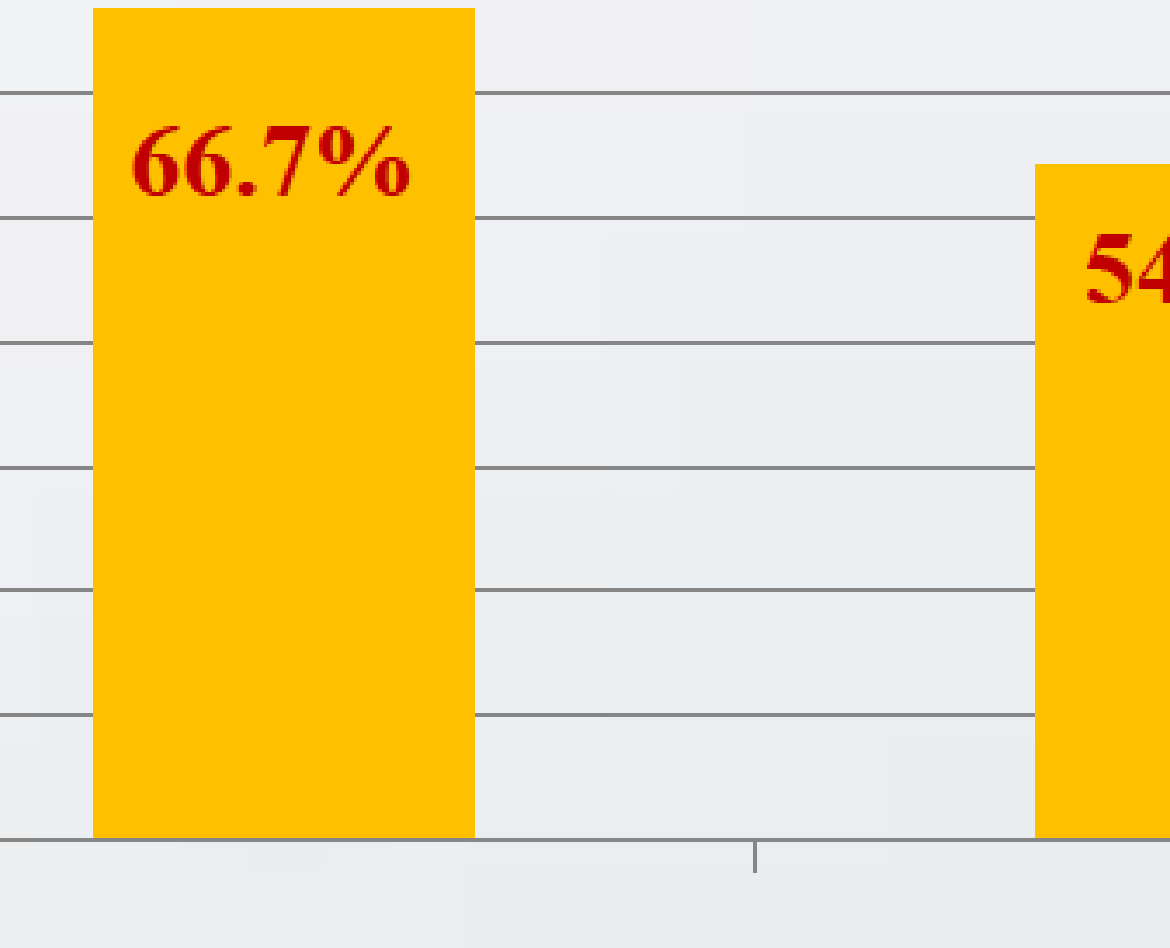

Episodic tachycardia Episodic elevations of blood pressure

\section{Scintigraphy with iodine-123-} metaiodobenzylguanidine

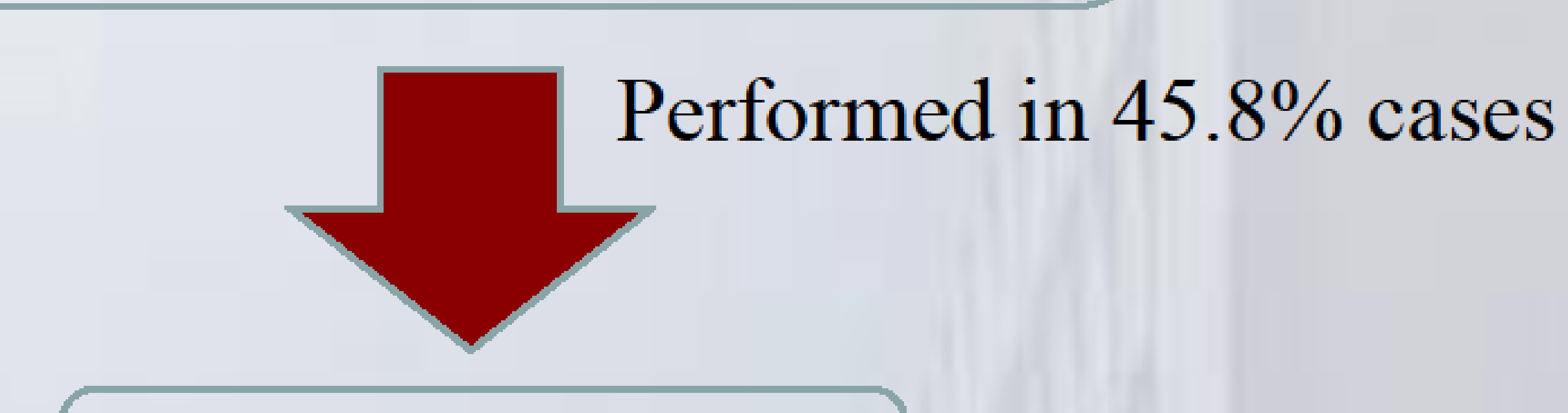

\section{POSITIVE}

Treatment:

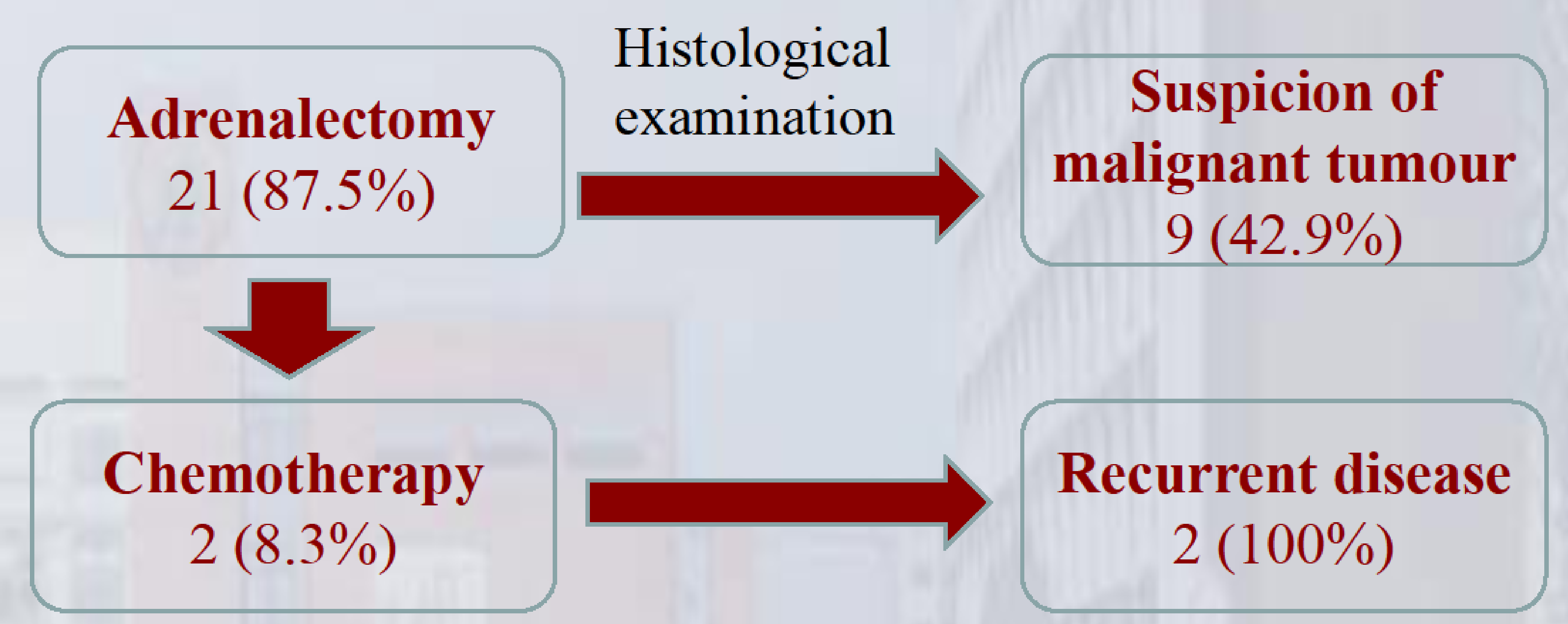

\section{Conclusions}

The incidence of pheochromocytoma in our hospital is $3,4 \%$ and meets the rates estimated by others. High prevalence of asymptomatic patients and suspicion of malignant tumors after histology urge for careful assessment of all adrenal masses for pheochromocytoma.

2. Terzolo M, Stigliano A, Chiodini I, Loli P, Furlani L, Arnaldi G, Reimondo G, Pia A, Toscano V, Zini M, Borretta G, Papini E, Garofalo P, Allolio B, Dupas B, Mantero F, Tabarin A. AME position statement on adrenal incidentaloma. Eur J Endocrinol. 2011 Jun;164(6):851-70.

3. Zeiger MA, Thompson GB, Duh QY, Hamrahian AH, Angelos P, Elaraj D, Fishman E, Kharlip J. AACE/AAES Adrenal Incidentaloma Guidelines, Endocr Pract. 2009 ;15. 